\title{
Late surgical correction of anomalous left coronary artery from pulmonary trunk in children, using autogenous aortic and pulmonary endotheliazed tube
}

\author{
Miguel A. Maluf \\ Cardiovascular Division, Universidade Federal de São Paulo (UNIFESP), São Paulo, Brazil \\ Email: miguelmaluf@gmail.com
}

Received 12 March 2012; revised 11 April 2012; accepted 5 June 2012

\begin{abstract}
Background: Direct re-implantation of an anomalous left coronary artery into the aorta is the preferred surgical option for creating a dual coronary arterial system in patients in whom the anomalous artery originated from the pulmonary trunk. This technique, however, is applicable only when the anomalous artery arises from the left posterior pulmonary sinus. We report the successfully late follow-up of series of patients employing a new technique using combined autogenous aortic and pulmonary endotheliazed tube. Patients and Methods: We have treated 3 patients, aged 2 months, 6 months and 8 months respectively, who presented with anomalous origin of the left coronary artery from the left posterior pulmonary sinus and moderated mitral valve insufficiency by left ventricle dysfunction. We used a trapdoor like coronary artery elongation with autogenous aortic and pulmonary endotheliazed tube to connect the left coronary ostium to ascending aorta, avoiding the mitral valve intervention. Results: There was no early or late death. All patients are in functional class $I$, with good biventricular function and competent mitral valve at a median follow-up of 94 months, ranged from 108 to 132 months. Postoperative Computerized tomogramphy of aorta in our $1^{\text {st }}$ patient showed good arterial flow, without any distortion. A local and distal stenosis of the left pulmonary artery was observed and submitted to stent treatment. Conclusions: The potential benefits of the trapdoor like and its modification technique are excellent operative exposure. The use of autogenous endothelized tube is a viable tissue capable of further growth, avoidance of injury to the aortic and pulmonary valvar apparatus or production of obstruction within the right ventricular outflow tract.
\end{abstract}

Keywords: Anomalous; Coronary Artery; Autogenous; Endotheliazed Tube; Surgery; Re-Implantation

\section{INTRODUCTION}

Anomalous origin of the left coronary artery (AL-CAPA) is a rare congenital heart disease. This anomaly leads to a coronary blood low flow and myocardial dysfunction by profoundly compromised of the left ventricle (LV). The optimal surgical management of such cases is not clearly established.

Direct re-implantation of an anomalous left coronary artery into the aorta is the preferred surgical option for creating a dual coronary arterial system in patients in whom the anomalous artery originated from the pulmonary trunk.

We report the successfully late follow-up of tree patients employing a new technique using combined autogenous aortic and pulmonary endotheliazed tube.

\section{PATIENTS AND METHOD}

We have treated 3 patients, aged 2 months, 6 months and 8 months, admitted in 2001, 2002 and 2004 respectively, at the Department of Pediatric Cardiology of São Paulo Federal University, who presented with anomalous origin of the left coronary artery from the left posterior pulmonary sinus.

All three patients presented at admission with dilated left ventricular cardiomyopathy, must undergo exclusion of ALCAPA. The electrocardiographic study evidence of left ventricular ischemia. The Dopplerechocardiogram detected mitral valve regurgitation and left ventricular function indices depressed. In two patients was possible to detect the left coronary artery origin from the left posterior sinus of the pulmonary rout. The evidences of flow from anomalous coronary to pulmonary artery, by color Doppler, is no confirmed in all cases. The cardiac catheterization was dispensed because carries a high risk in this group of the patients.

\section{Surgical Procedure}

We used our proposed technique: trapdoor like coro- 
nary artery elongation [1], in all 3 cases the left coronary artery origin from left posterior sinus of the pulmonary artery was transfer to the aortic rout. The first patient, a 2 months old, masculine boy, was operated in March 2001.

The operation was performed during the operative approach was observed an important artery (Figure 1) and presented fibrous area in the left ventricle apex (Figure 2).

After the cardiopulmonary bypass (CPB) was installed with moderated hypothermia, the aortic was clamped and blood cardioplegic solution was administered. The pulmonary trunk was sectioned, two longer flaps were tailored from: the anterior wall of the pulmonary artery, and the left lateral aortic wall (Figure 3).

To construct an endotheliazed conduit, the aortic and pulmonary flaps were sutured by the lateral border, performed an arterial tube and maintaining the primitive coronary ostium of the left coronary artery, in situ (Figure 4). Finally the PA was reconstructed using a bovine pericardium graft. The mitral valve was not addressed in any case.

\section{RESULTS}

There was no early or late death. All patients, at 7, 9 and 11 years old, are in functional class I (NYHA), with good biventricular function and competent mitral valve at a median follow-up of 94 months, with a range from 108

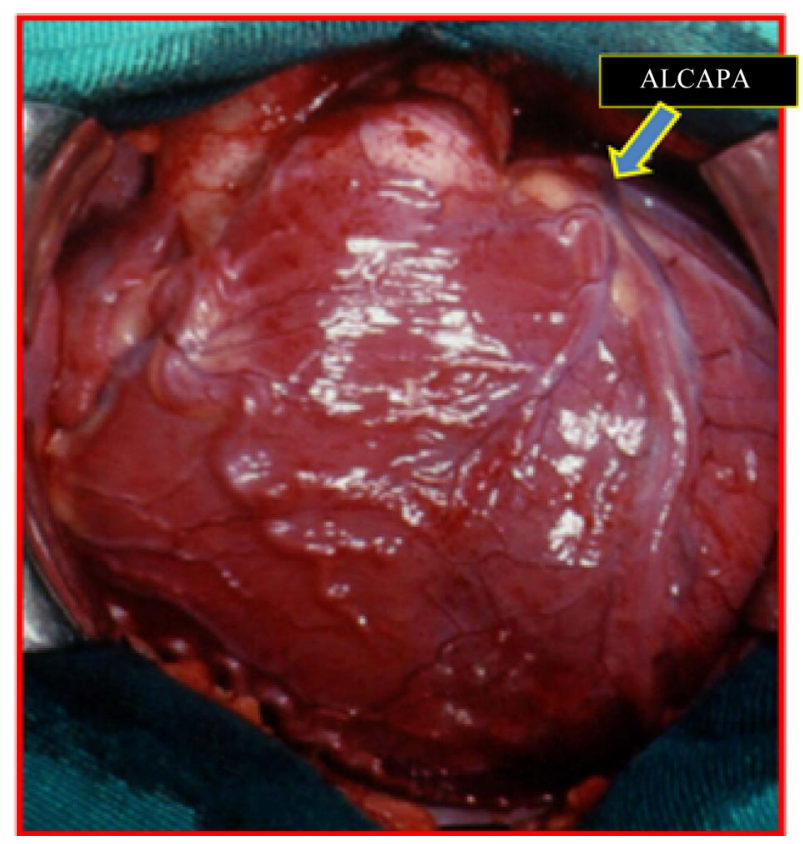

Figure 1. Surgical image ( $1^{\text {st }}$ patient). External appearance of the heart, we observed the anomalous origin of left coronary artery from the pulmonary artery (ALCAPA) and significant intercoronary shunt.
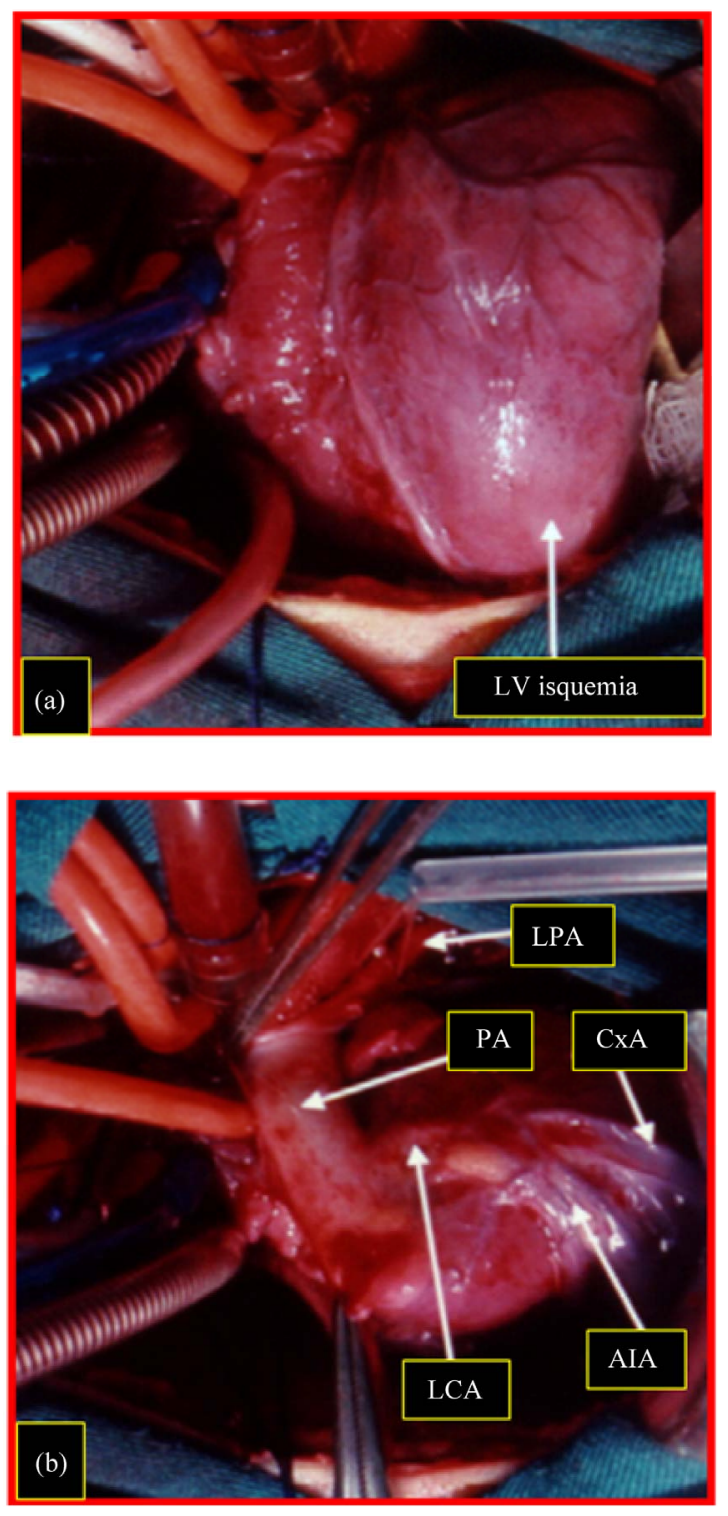

Figure 2. Surgical image ( $1^{\text {st }}$ patient). Patient on cardiopulmonary bypass. (a) Aspect of the ischemic anteroapical region of the left ventricle; (b) Origin of the left coronary artery from the left posterior sinus of the pulmonary valve. LV: left ventricle; LCA: left coronary artery; PA: pulmonary artery; LPA: left pulmonary artery; The Cx: circumflex artery; EIA: anterior interventricular artery.

to 132 months.

Postoperative clinical examination of our $1^{\text {st }}$ patient (8 years old) showed: regular heart rhythm absence cardiac murmur; normal ECG and Doppler echocardiogram showed preserved left ventricular function, mitral valve competence and patent arterial conduit from the ascending aorta to left coronary artery (Figure 5).

Computadorized Tomography images of this $1^{\text {st }}$ patient showed implantation of autogenous arterial conduit in the ascending aorta above the aortic sinus, with 


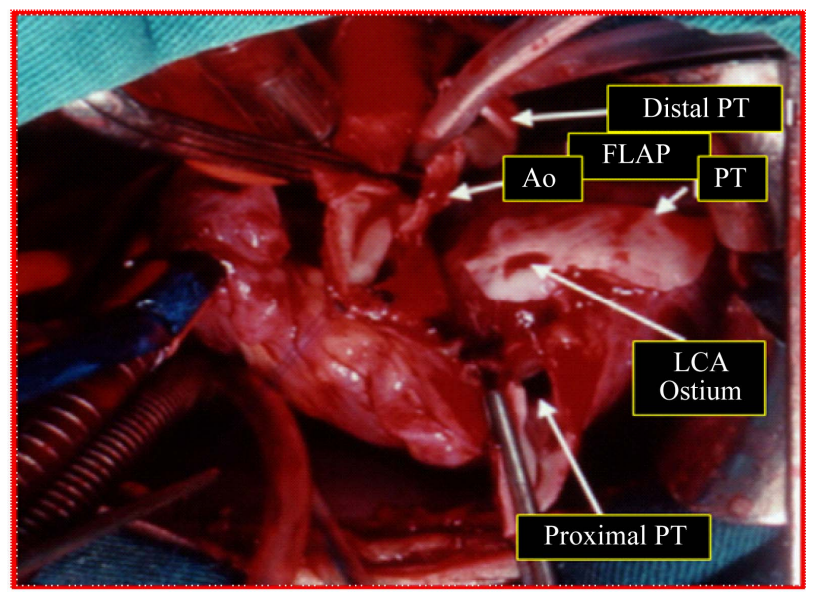

Figure 3. Surgical image ( $1^{\text {st }}$ patient). Patient on cardiopulmonary bypass. After section of the pulmonary trunk (PT), was performed the resection of the ostium of the left coronary artery (LCA) along with flap from the wall of the PT. Another pedicled flap was made on the lateral wall of the aorta (Ao).

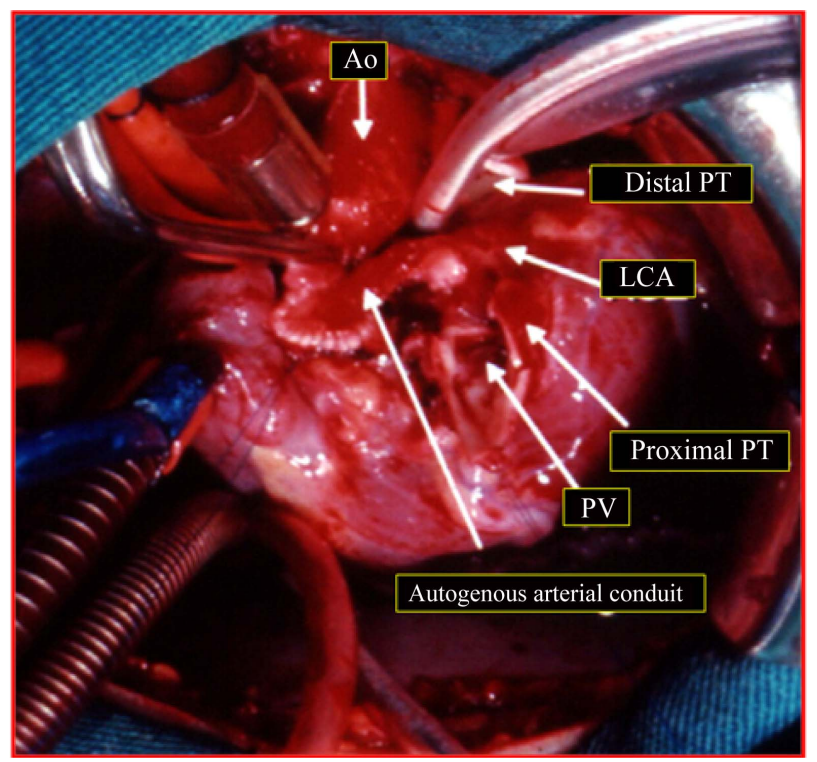

Figure 4. Surgical image ( $1^{\text {st }}$ patient). Patient on cardiopulmonary bypass. Implantation technique of the left coronary artery (LCA) in the ascending aorta (Ao). Manufacture of autogenous arterial conduit with flaps of pulmonary artery and aorta, sutured by their edges and side extremity anastomosed to the ascending aorta. PV: pulmonary valve; PT: pulmonary trunk.

good arterial flow and uniform diameter without any distortion (Figures 6(a) and (b)); reconstruction of the pulmonary artery (Figure 7(a)) and the presence of a significant stenosis on the distal left pulmonary artery not detected in prior pre- and postoperative exams (Figure 7(b)).

With 9 years old, this patient was submitted to angiographic study for dilatation of pulmonary stenosis and pulmonary stent graft implanted (Figures 8(a)-

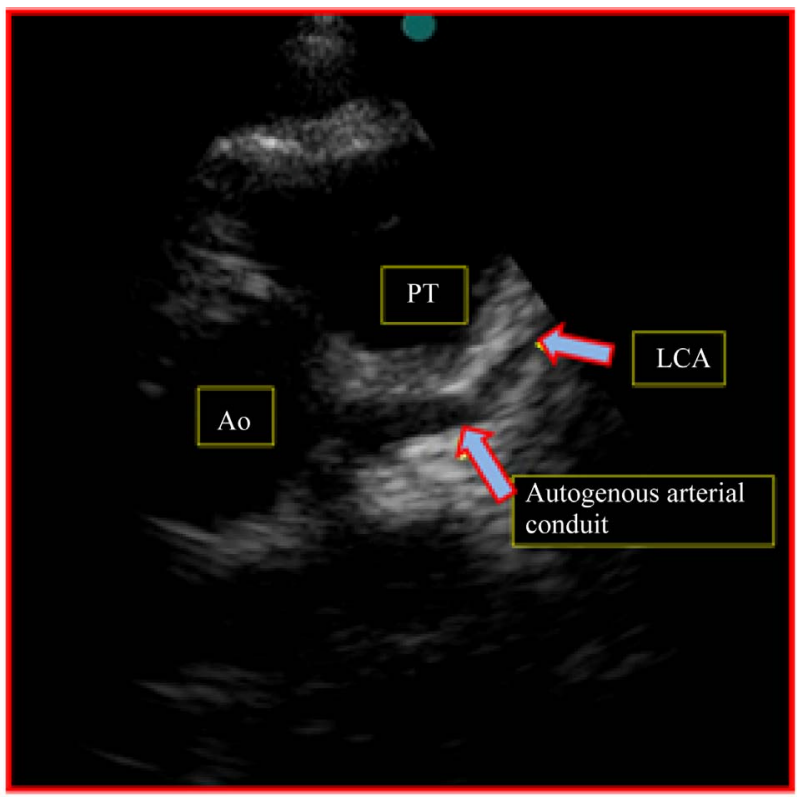

Figure 5. Follow-up $8^{\text {th }}$ year $\left(1^{\text {st }}\right.$ patient). Doppler echo- cardiography postoperative image, observed autogenous arterial conduit connecting the aorta to the left coronary artery, patent, with uniform diameter. PT: pulmonary trunk; LCA: left coronary artery; Ao: aorta.

(c)).

\section{DISCUSSION}

ALCAPA is a rare cardiac malformation described by Bland-White-Garland in 1933 and accompanied by high mortality in infants; Vouhe et al. [1] consider the mortality of about $90 \%$ in the first year of life.

This is a congenital defect where the myocardial function was deeply compromised due to myocardial ischemia and as it occurred in ours patients, it began at a young age, probably immediately after the closure of the patent ductus arteriosus.

The most common site of origin of the left coronary artery, as in ours patients, occurs within the left posterior pulmonary valve, standing away from the ascending aorta in any attempt for direct reimplantation into the aorta.

During the external examination of the heart, we observed the presence of the main coronary arteries and their branches dilated and tortuous, the result of coronary shunt right to left (Figure 1).

The purpose of correction of ALCAPA is to create two separate coronary systems, however, the location of the left coronary ostium near or distant from the aorta, has raised interest and stimulated the creativity of the cardiac surgeon, to develop new techniques and improve outcomes in the middle and long term.

The direct reimplantation of the left coronary artery on the ascending aorta involves the dissection and move- 

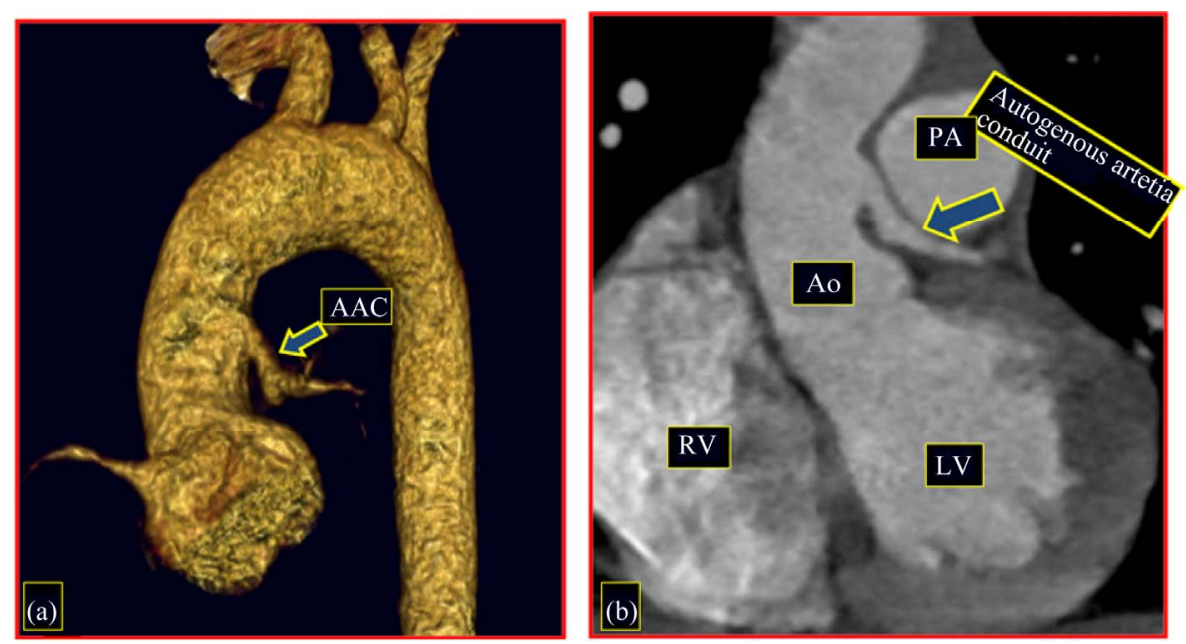

Figure 6. Follow-up $8^{\text {th }}$ year $\left(1^{\text {st }}\right.$ patient). Image of CT angiography, postoperative. (a) Implantation of autogenous arterial cunduit in the ascending aorta above the aortic sinus; (b) Autogenous arterial conduit patency, with uniform diameter. AAC: autogenous arterial conduit; RV: right ventricle; LV: left ventricle; PA: pulmonary artery; Ao: aorta.
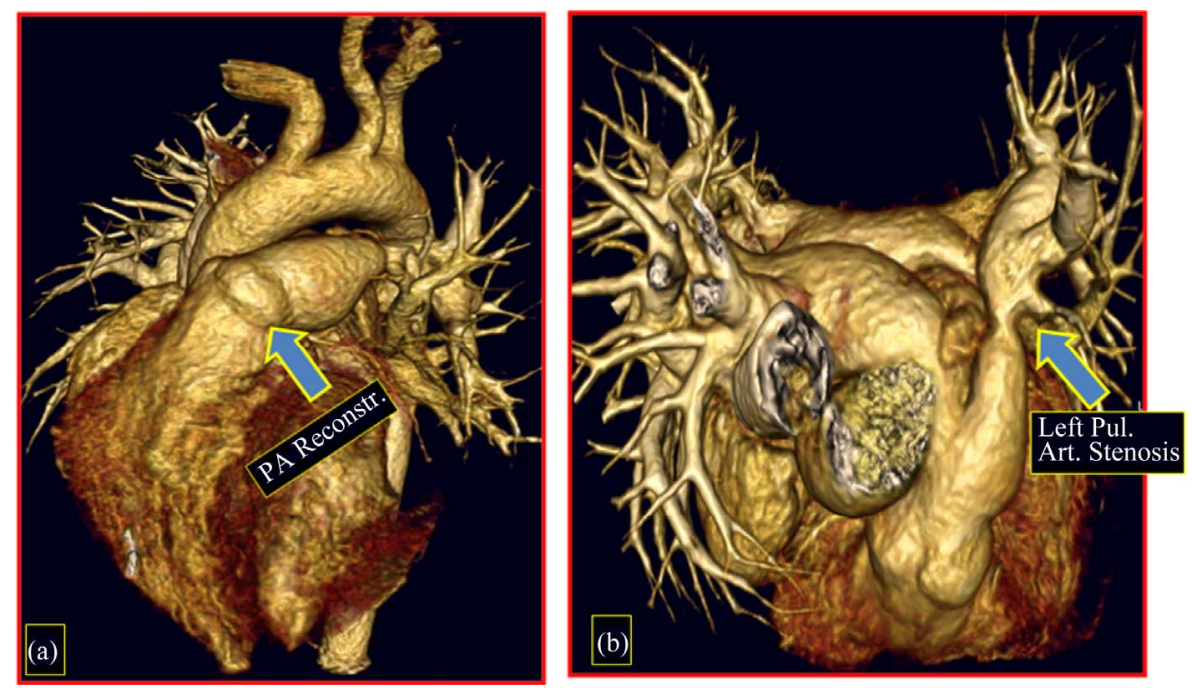

Figure 7. Follow-up $8^{\text {th }}$ year ( $1^{\text {st }}$ patient). Image of CT angiography, postoperative. (a) Reconstruction of the pulmonary artery; (b) Image of stenosis located in the distal left pulmonary artery. PA: pulmonary artery.

ment of the coronary artery trunk and its main branches, but tension on the sutures compromise coronary flow and result in high mortality. In our series of 17 patients with ALCAPA, we had two deaths due to ischemic dysfunction of the $\mathrm{LV}$, probably due to tension in the anastomosis after aortic implantation of the left coronary artery, which originated within the left posterior sinus of the pulmonary valve.

Aiming to avoid such incidents and to improve the operative late outcome, this technique has been modified and now employs procedures to lengthen the proximal coronary artery and permit implantation in the aorta without tension.

Thus, Sese et al. [2] in 1992 described a new tech- nique of stretching of the coronary artery, using a flap of the pulmonary artery wall and a pedicled flap of the aortic wall; this technique was called "trap-door". The criticism of this procedure is still the need for dissection of the main branches of the coronary artery, for its approach to the ascending aorta, often hindered by the presence of tortuous coronary collaterals.

Similar procedures have been published by Tashiro et al. [3] in 1992 and Von Son et al. [4] in 1997. Katsumata et al. [5] in 1999 and Murthy et al. [6] in 2001, based on initial experience stretching the left coronary artery, now with aortic and pulmonary flaps, larger in width and length, built autogenous arterial conduits or tubs, to reimplant the left coronary artery originating from the 


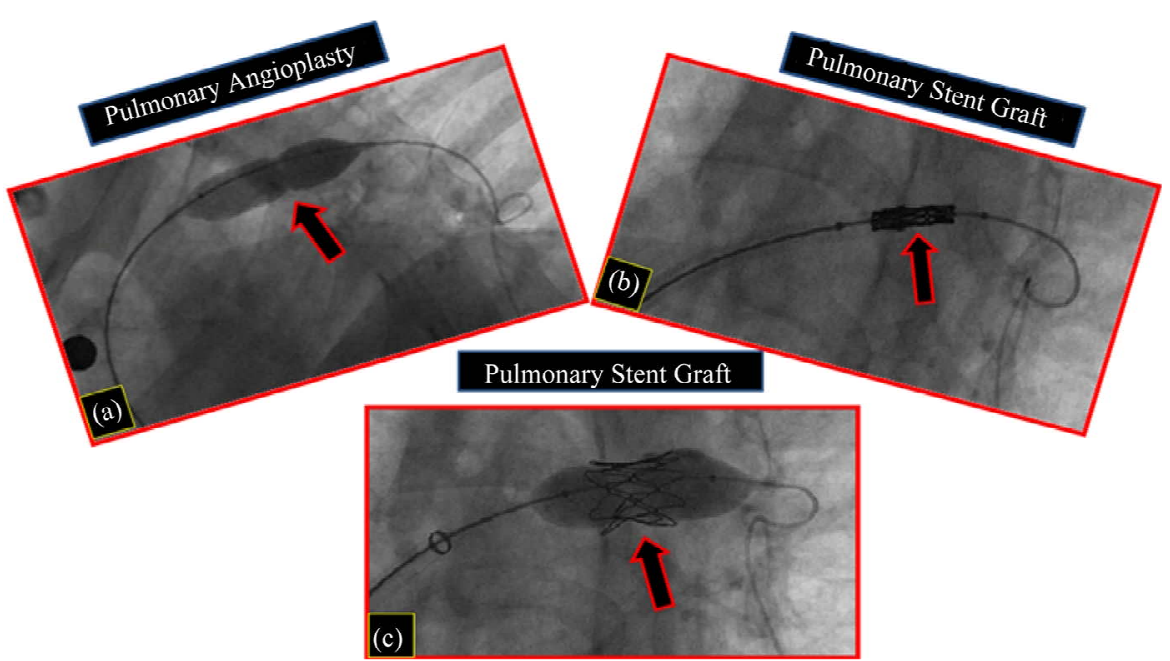

Figure 8. Follow-up $9^{\text {th }}$ year $\left(1^{\text {st }}\right.$ patient). Postoperative cardiac catheterization. Cineangiographic image. (a) Pulmonary angioplasty with balloon; (b) Pulmonary stent implanted; c-Pulmonary stent dilatation, with balloon.

left posterior sinus of the pulmonary valve without tension and without dissection of the main coronary branches.

The experience of our group of congenital heart diseases at Unifesp started successfully in December 2001 and published three years after, the results of the 1st case operated employing this new technique (Maluf et al. [7]).

At late follow-up (mean: 94 months), all three patients are in FC I (NYHA) and remained asymptomatic during physical activity and without medication. The examinations of CT angiography, performed each 2 years, certify the successful outcome of this technique, showing the autologous arterial graft patency and uniform diameter throughout its length.

Mitral regurgitation is a common finding in patients with ALCAPA, caused by ischemic papillary muscles and LV dilation. No cases of our series had mitral valve surgery approach, being aware that after improvement of the ventricular function, there would be regression of mitral valve dysfunction, as documented in ours patients. This conservative approach has been adopted by Vohue et al. [1], with improvement of mitral dysfunction. The authors report that mitral valve repair in infants is a procedure without very favorable results, while the conservative approach does not increase surgical risk.

\section{CONCLUSIONS}

1) The coronary artery transference was technically safe and feasible.

2) The endotheliazed inner conduit remains pervious during the late follow-up.

3) Long-term clinical outcome and left ventricular function are good despite severe left ventricular dysfunction at presentation.

4) Mitral valve repair was not necessary. Competent mitral valve was observed after the return of the normal left ventricle function.

\section{REFERENCES}

[1] Vohue, P.R., Tamisier, D., Sidi, D., Vernant, F., Mauriat, P., Pouard, P. and Leca, F. (1992) Anomalous left coronary artery from the pulmonary artery: Results of isolated aortic reimplantation. Annals of Thoracic Surgery, 54, 621-627. doi:10.1016/0003-4975(92)91004-S

[2] Sese, A. and Omoto, Y. (1992) New technique in the transfer of an anomalously originated left coronary artery to the aorta. Annals of Thoracic Surgery, 53, 527-529. doi:10.1016/0003-4975(92)90290-K

[3] Tashiro, T., Todo, K., Hamta, Y., Yasunaga, H., Nagata, M. and Nakamura, M. (1993) Anomalous origin of the left coronary artery from the pulmonary artery: New operative technique. Journal of Thoracic and Cardiovascular Surgery, 106, 718-722.

[4] Von Son, J.A. and Mohr, F.W. (1997) Repair of anomalous connection of the left coronary artery to the pulmonary artery using native aorta and pumnonary tissue flaps. European Journal Cardio-Thoracic Surgery, 12, 322-329. doi:10.1016/S1010-7940(97)00124-3

[5] Katsumata, T. and Westaby, S. (1999) Anomalous left coronary artery from the pulmonary artery: A simple method for aortic implantation with autogenous arterial tissue. Annals of Thoracic Surgery, 68, 1090-1011. doi:10.1016/S0003-4975(99)00761-4

[6] Murthy, K.S., Krishnanaik, S., Mohanty, S.R., Varghese, R. and Cherian, K.M. (2001) A new repair for anomalous left coronary artery. Annals of Thoracic Surgery, 71, 13841386. doi:10.1016/S0003-4975(00)02506-6

[7] Maluf, M.A., Mangia, C.M., Diogenes, M.S., Carvalho, A.C. and Buffolo, E. (2004) Anomalous coronary artery from pulmonary artery: Autogenous arterial tube for aortic implantation. Journal of Cardiovascular Surgery, 45, 577-579. 